\title{
Snail Protein Expression as a Hallmark of Gastric Carcinoma in Biopsy Samples
}

\author{
Hiroyuki Tanishima, Ting Gui, Yujing Sun, Aiko Shimokado, \\ Takashi Ozaki, and Yasuteru Muragaki \\ First Department of Pathology, School of Medicine, Wakayama Medical University, 811-1 Kimiidera, Wakayama 641-0011, Japan \\ Correspondence should be addressed to Yasuteru Muragaki,ymuragak@wakayama-med.ac.jp
}

Received 31 March 2012; Accepted 11 June 2012

Academic Editors: J. A. Jimenez-Heffernan, G. Valente, and A. Wincewicz

Copyright (C) 2012 Hiroyuki Tanishima et al. This is an open access article distributed under the Creative Commons Attribution License, which permits unrestricted use, distribution, and reproduction in any medium, provided the original work is properly cited.

Overexpression of the Snail gene transcriptional repressor promotes an epithelial-to-mesenchymal transition (EMT) in epithelial tumor cell lines. In this study, we aimed to determine the correlation between Snail protein expression and clinicopathological features and to test whether Snail can be used as a marker to distinguish gastric carcinomas from benign tissues in biopsy samples. The results of immunohistochemistry with an antibody against Snail showed that most adenocarcinomas had positive Snail expression, whereas weak Snail expression was detected in a small number of gastritis and gastric adenomas. Snail-positive cells were detected in the stroma as well as in the glandular epithelium in some adenocarcinomas. In addition to Snail immunostaining, immunostaining of the EMT-related molecules, E-cadherin and vimentin, was performed. E-cadherin was not detected in adenocarcinomas that expressed Snail, whereas gastritis and adenomas stained positively for E-cadherin. Vimentin expression was seen in adenocarcinomas with positive Snail expression, whereas gastritis and adenomas did not express vimentin. In conclusion, we propose that Snail is a useful biomarker to distinguish gastric adenocarcinomas from benign lesions in biopsy samples.

\section{Introduction}

The transcription factor Snail is a zinc finger protein that represses transcription of the E-cadherin gene through an interaction of the Snail $\mathrm{COOH}$-terminal region with a $5^{\prime}-$ CACCTG- $3^{\prime}$ sequence called the E-box in the E-cadherin promoter region [1]. The loss of E-cadherin expression has been shown to be responsible for the loss of cell-cell adhesion and cell polarity that occurs during the early stages of invasion and metastasis of carcinoma cells [1]. During the process of tumor invasion, tumor cells lose their properties of cell-cell adhesion and frequently undergo profound changes in their phenotype known as epithelial-to-mesenchymal transition (EMT) [2]. Snail is one of the most important regulators of EMT [3].

Gastric cancer is the most common malignant tumor in Japan. In 1998, more than 100,000 new cases were reported. By 2015, it is anticipated that this number will climb to nearly 150,000 [4]. The only potentially curative treatment for gastric cancer is surgical resection of all gross and microscopic disease. Deciding which type of surgery to perform is one of the most important surgical issues in this disease. Recent advances in endoscopy have allowed easy exploration of the stomach by gastroenterologists. However, the final diagnosis of gastric cancer and the definitive treatment still consist of surgical intervention [5].

Overexpression of Snail is associated with metastasis and poor prognosis in gastric cancer [6]. However, in most of these studies, Snail expression was analyzed with surgically resected specimens. In the present study, we examined the difference in the immunoreactivity of Snail protein in biopsy specimens of gastric mucosa and showed that Snail can play a significant role in the diagnosis of gastric cancer.

\section{Materials and Methods}

2.1. Samples. Two hundred forty-nine gastric endoscopy specimens, including 43 gastritis, 41 adenoma, and 165 carcinoma specimens, were obtained from the First Department 
of Pathology, Wakayama Medical University. Clinicopathological parameters, such as age, sex, histological subtype, and tumor location, were evaluated by reviewing pathological records. These data are shown in Tables 1, 2, and 3, for gastritis, adenomas, and adenocarcinomas, respectively. Of 165 specimens diagnosed as gastric cancer, 36 (22\%) specimens were well differentiated tubular adenocarcinoma, 48 (29\%) moderately differentiated tubular adenocarcinoma, $51(31 \%)$ poorly differentiated adenocarcinoma, and 30 (18\%) signet-ring cell carcinoma, according to the Japanese classification of gastric carcinoma (Japanese Gastric Cancer association, 2010) [7]. The protocol for the research project has been approved by the Ethics Committee of Wakayama Medical University.

2.2. Immunohistochemical Staining. Immunohistochemical staining was performed on $4-\mu \mathrm{m}$ sections of biopsy samples, which were fixed with $4 \%$ formalin and embedded in paraffin. The sections were incubated with an anti-Snail polyclonal antibody (1:500, Abcam, Cambridge, UK), an anti-E-cadherin monoclonal antibody (clone DECMA-1, $1: 800$, Sigma-Aldrich, St. Louis, USA), or an antivimentin monoclonal antibody (clone V9, 1 : 100, Dako Cytomation, Glostrup, Denmark) overnight at $4^{\circ} \mathrm{C}$. An EnVison HRP stain system (Dako Cytomation) was used for visualization with hematoxylin counterstain according to the manufacturer's instructions.

Immunohistochemical evaluations were conducted by two investigators blinded to the clinicopathological data. Snail staining was graded as positive only when nuclear staining was detectable, as described previously for colon cancer [2]. When the number of cells with positive nuclear staining in a sample was lower than $1 \%$, the sample was considered negative for Snail. For the semiquantitative evaluation, Snail staining was scored according to the nuclear staining on a scale of 0 to 300 , where the number is given by multiplying the percentage of positive cells (from 0 to $100 \%$ ) and the intensity of immunoreactivity (1 to 3 ). These samples were categorized into three groups based on the score: negative expression, <10; low expression, 10-20; high expression, $>20$.

To evaluate E-cadherin and vimentin staining, cells with positive staining were counted. When more than $10 \%$ of cells were positive, the sample was considered positive.

2.3. Statistical Analysis. Differences were compared using Spearman's $\chi^{2}$ test for qualitative variables. All statistical tests were two-sided, and statistical significance was set at the $P<$ 0.05 level. All analyses were performed using SPSS, version 13.0 (SPSS Inc. Chicago, USA).

\section{Results}

3.1. Snail Protein Expression Is Correlated with Gastric Adenocarcinoma. Snail contributes to the initiation of classical EMT [1]. To determine whether Snail expression was correlated with histological types in gastric biopsy tissues,
TABLE 1: Characteristics of 43 patients with gastritis.

\begin{tabular}{lc}
\hline Characteristic & $N(\%)$ \\
\hline Age, mean $( \pm$ SD) & $64.57 \pm 15.3$ \\
Sex & \\
$\quad$ Female & $28(65 \%)$ \\
$\quad$ Male & $15(35 \%)$ \\
Tumor location & \\
Anastomosis & $2(4.6 \%)$ \\
EC & 0 \\
CF & $16(37.2 \%)$ \\
LC & $9(20.9 \%)$ \\
GC & $6(14.0 \%)$ \\
AP & $10(23.3 \%)$ \\
Snail expression & \\
Negative & $39(91 \%)$ \\
Low & $4(9 \%)$ \\
\hline Total & 43 \\
\hline
\end{tabular}

(AP: Antropyloric region; LC: lesser curvature; GC: greater curvature;CF: Corpus Fornix; EC: eso-cardial region).

TABLE 2: Characteristics of 41 patients with gastric adenoma.

\begin{tabular}{lc}
\hline Characteristic & $N(\%)$ \\
\hline Age, mean $( \pm$ SD $)$ & $72.31 \pm 15.62$ \\
Sex & \\
$\quad$ Female & $23(56 \%)$ \\
$\quad$ Male & $18(44 \%)$ \\
Tumor location & \\
Anastomosis & $2(4.8 \%)$ \\
EC & 0 \\
CF & $17(41.5 \%)$ \\
LC & $10(24.4 \%)$ \\
GC & $7(17.1 \%)$ \\
AP & $5(12.2 \%)$ \\
Snail expression & $34(83 \%)$ \\
Negative & $7(17 \%)$ \\
Low & 41 \\
\hline Total & \\
\hline
\end{tabular}

immunohistochemistry (IHC) with Snail antibodies was performed on biopsy sections.

IHC analysis for Snail expression revealed that most samples with gastritis and adenoma showed negative staining for Snail, although focal and low expression was observed (Figure 1(a), Tables 1 and 2). There were no correlations between Snail expression and age, sex, or lesion location.

Table 3 summarizes the clinicopathological parameters of well differentiated tubular adenocarcinomas, moderately differentiated tubular adenocarcinomas, poorly differentiated adenocarcinomas, and signet-ring cell carcinomas. Most carcinoma patients were over 50 years in age. Only three cases of poorly differentiated adenocarcinomas were seen in patients under 50 years in age. Most gastric carcinomas occurred in the corpus and fornix (CF), although the 


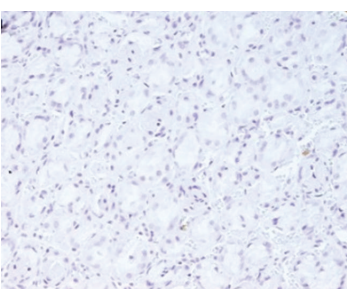

(a)

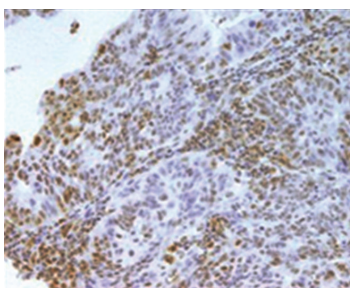

(d)

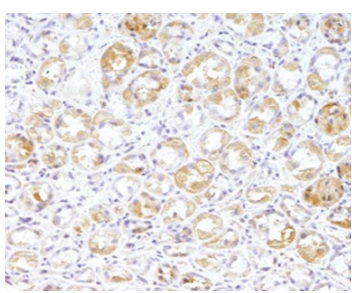

(a)

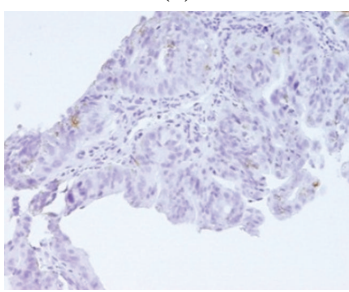

(d)

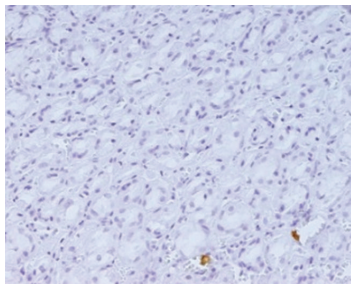

(a)

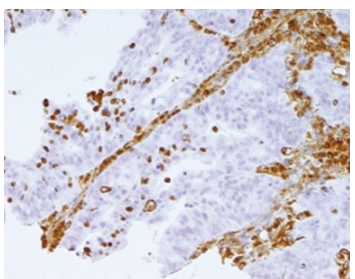

(d)

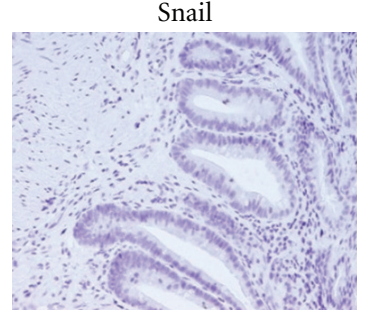

(b)

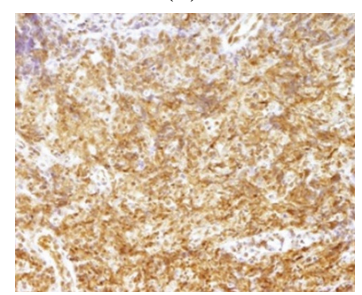

(e)

(A)

E-cadherin

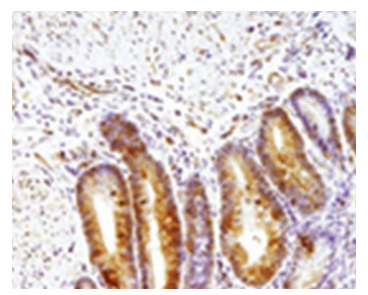

(b)

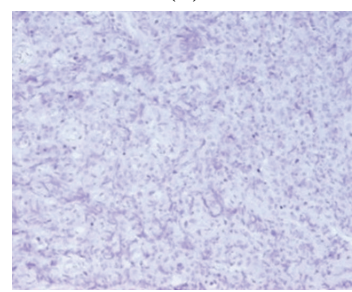

(e)

(B)

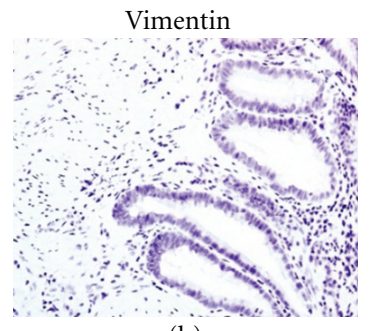

(b)

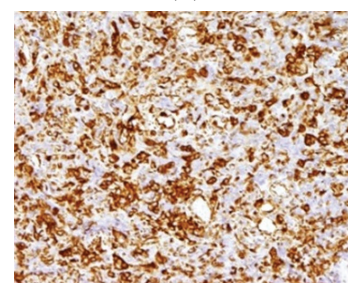

(e)

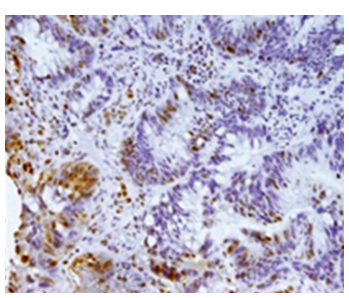

(c)

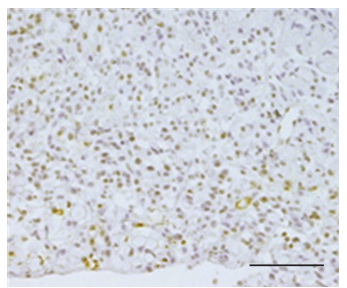

(f)

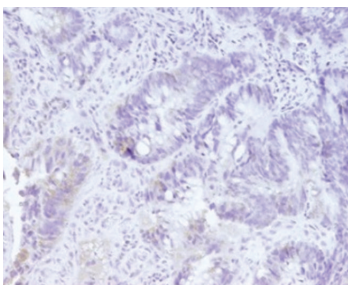

(c)

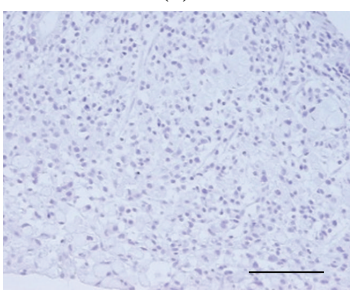

(f)

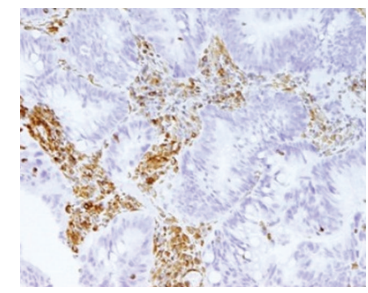

(c)

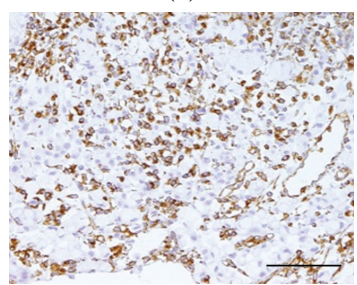

(f)

(C)

FIGURE 1: Representative pictures for immunohistochemistry of Snail (A), E-cadherin (B), and vimentin (C) in biopsy samples from gastritis, adenomas, and adenocarcinomas. Sections were stained with antibodies against Snail (A), E-cadherin (B), and vimentin (C). Consecutive sections were used from patients with gastritis (a), adenoma (b), well differentiated tubular adenocarcinoma (c), moderately differentiated tubular adenocarcinoma (d), poorly differentiated adenocarcinoma (e), and signet-ring cell carcinoma (f). Scale bar: $100 \mu \mathrm{m}$. 
TABLE 3: Characteristics of 165 patients with gastric carcinoma.

\begin{tabular}{lcccc}
\hline Characteristic & Well & Moderate & Poorly & Signet-ring cell \\
\hline $\begin{array}{l}\text { Age, mean }( \pm \text { SD) } \\
\text { Sex }\end{array}$ & $69.35( \pm 11.8)$ & $71.57( \pm 12.0)$ & $67.52( \pm 11.9)$ & \\
$\quad$ Female & $19(53 \%)$ & $11(23 \%)$ & $33(65 \%)$ & $16(53 \%)$ \\
$\quad$ Male & $17(47 \%)$ & $37(77 \%)$ & $18(35 \%)$ & $14(47 \%)$ \\
Tumor location & & & & \\
Anastomosis & $2(5.6 \%)$ & 0 & $2(3.9 \%)$ & 0 \\
EC & $4(11.1 \%)$ & $2(4.2 \%)$ & $19(37.3 \%)$ & 0 \\
CF & $14(38.9 \%)$ & $24(50 \%)$ & $7(13.7 \%)$ & $11(37 \%)$ \\
LC & $7(19.4 \%)$ & $4(8.3 \%)$ & $9(17.6 \%)$ & $7(23 \%)$ \\
GC & $5(13.9 \%)$ & $6(12.5 \%)$ & $14(27.5 \%)$ & 0 \\
AP & $4(11.1 \%)$ & $12(25 \%)$ & 51 & 30 \\
\hline Total & 36 & 48 & \\
\hline
\end{tabular}

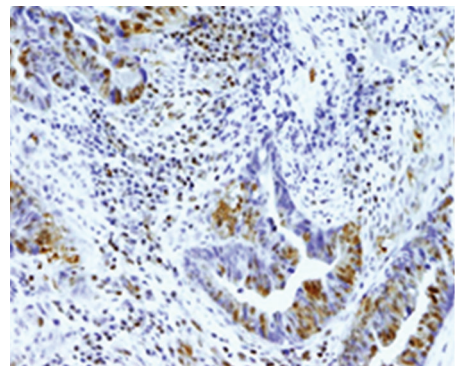

(a)

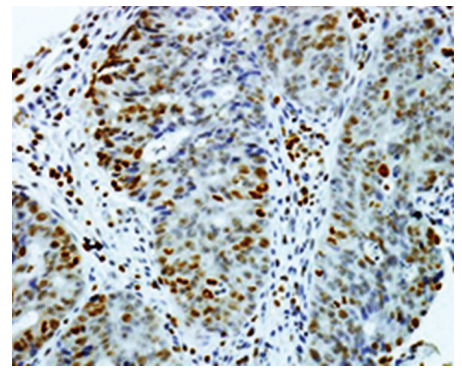

(b)

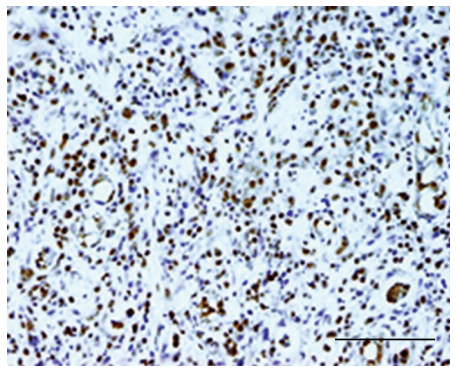

(c)

FIGURE 2: Representative pictures for immunohistochemistry of Snail showing positive cells in the stroma and glandular cells in well differentiated (a) and moderately differentiated (b) tubular adenocarcinomas as well as in a poorly differentiated adenocarcinoma (c). Scale bar: $100 \mu \mathrm{m}$.

antropyloric region (AP) was the most frequent location for moderately differentiated tubular adenocarcinomas and poorly differentiated adenocarcinomas $(25 \%$ and $27.5 \%$, resp.; see Table 3 ).

The relationship between Snail expression and clinicopathological parameters for adenocarcinomas is shown in Table 4. Spearman correlation statistics did not show any significant correlation between tumor location and Snail staining $(P=0.381)$. The ratio of male to female carcinoma patients was 1.09; sex was not significantly correlated with Snail staining rates $(P=0.771)$.

Positive Snail expression was observed in $97.2 \%$ of well differentiated tubular adenocarcinomas, all moderately differentiated tubular adenocarcinomas, $88.9 \%$ of poorly differentiated carcinomas, and $70 \%$ of signet-ring cell carcinomas. There was no significant correlation between Snail staining and degree of carcinoma differentiation $(P=0.362)$ (Table 4$): 72.2 \%(26 / 36)$ of well differentiated tubular adenocarcinomas, $72.9 \%(35 / 48)$ of moderately differentiated tubular adenocarcinomas, and $86.3 \%$ (44/51) of poorly differentiated adenocarcinomas, cells showed diffuse and strong nuclear staining for Snail, whereas a lower percentage of signet-ring cell carcinomas (36.7\% (11/30)) had high Snail expression (Table 4). Nine cases out of 30 signet-ring carcinomas were negative for Snail. In 56\% (76/135) of adenocarcinomas including well and moderately differentiated tubular adenocarcinomas as well as poorly differentiated adenocarcinomas, positive staining for Snail was observed in both types of carcinoma and stromal cells (Figure 2).

3.2. Vimentin and E-Cadherin Expression Was Closely Correlated with Snail Expression. All biopsy cases were stained with E-cadherin and vimentin antibodies.

E-cadherin and Snail expression levels were negatively correlated in patients with gastritis and adenoma $(P=0.024$, 0.034; Table 5). IHC with E-cadherin antibodies showed diffuse and strong positive staining in cell membrane of the foveolar and glandular epithelium in 79\% (34/43) of patients with gastritis and 61\% (25/41) of those of adenoma (Figure $1(\mathrm{~b}))$. By contrast, IHC was negative for E-cadherin in $73 \%$ $(121 / 165)$ of carcinomas. 101 out of the 121 cases stained positively for Snail, indicating that Snail and E-cadherin were also negatively correlated in gastric carcinoma (Figures 1(a) and 1 (b), and Table 6).

In all biopsy samples, vimentin and Snail expression were closely correlated. Gastritis and adenomas samples that were negative for Snail also showed weak or negative vimentin expression (33/43 and 23/41, resp.; Figures 1(a), and 1 (c) and Table 5). Vimentin showed strong expression in carcinoma cells as well as the stromal cells, especially in poorly differentiated adenocarcinomas (40/51) (Figure 1(c)), 
TABLE 4: Correlation between Snail expression and clinicopathological features of gastric carcinoma. Spearman's rank correlation was used to determine the statistical significance of the correlations between Snail expression and the pathological variables. SPSS software v. 16.0 for Windows was used for the statistical analysis.

\begin{tabular}{|c|c|c|c|c|c|}
\hline & \multicolumn{3}{|c|}{ Snail expression } & \multirow{2}{*}{ Total } & \multirow[t]{2}{*}{$P$} \\
\hline & Negative $(0 \sim 10)$ & Low $(10 \sim 20)$ & $\operatorname{High}(>20)$ & & \\
\hline Total & $10(6.1 \%)$ & $39(23.6 \%)$ & $116(70.3 \%)$ & 165 & \\
\hline Differentiation & & & & & 0.362 \\
\hline Well & $1(2.8 \%)$ & $9(25 \%)$ & $26(72.2 \%)$ & 36 & \\
\hline Moderately & 0 & $13(27.1 \%)$ & $35(72.9 \%)$ & 48 & \\
\hline Poorly & 0 & $7(13.7 \%)$ & $44(86.3 \%)$ & 51 & \\
\hline Signet-ring cell & $9(30 \%)$ & $10(33.3 \%)$ & $11(36.7 \%)$ & 30 & \\
\hline Sex & & & & & 0.771 \\
\hline Female & $6(7.6 \%)$ & $18(22.8 \%)$ & $55(69.6 \%)$ & 79 & \\
\hline Male & $4(4.7 \%)$ & $21(24.4 \%)$ & $61(70.9 \%)$ & 86 & \\
\hline Tumor location & & & & & 0.381 \\
\hline Anastomosis & 0 & 0 & $2(100 \%)$ & 2 & \\
\hline EC & 0 & $1(12.5 \%)$ & $7(87.5 \%)$ & 8 & \\
\hline $\mathrm{CF}$ & $5(7.4 \%)$ & $19(27.9 \%)$ & $44(64.7 \%)$ & 68 & \\
\hline LC & $3(10 \%)$ & $4(13.3 \%)$ & $23(76.7 \%)$ & 30 & \\
\hline GC & $2(7.4 \%)$ & $9(33.3 \%)$ & $16(59.3 \%)$ & 27 & \\
\hline $\mathrm{AP}$ & 0 & $6(20 \%)$ & $24(80 \%)$ & 30 & \\
\hline
\end{tabular}

TABLE 5: Correlation between Snail expression and E-cadherin, vimentin expression in gastritis, and adenoma biopsy samples.

\begin{tabular}{|c|c|c|c|c|c|c|c|c|}
\hline & \multicolumn{2}{|c|}{ Snail expression in gastritis } & \multirow{2}{*}{ Total } & \multirow{2}{*}{$P$} & \multicolumn{2}{|c|}{ Snail expression in adnoma } & \multirow{2}{*}{ Total } & \multirow{2}{*}{$P$} \\
\hline & Negative $(<10)$ & Low (10-20) & & & Negative $(<10)$ & Low (10-20) & & \\
\hline E-cadherin & & & 43 & 0.024 & & & 41 & 0.034 \\
\hline Negative $(0-10 \%)$ & $7(16 \%)$ & $3(6 \%)$ & 10 & & $4(10 \%)$ & $12(30 \%)$ & 16 & \\
\hline Positive (>10\%) & $32(74 \%)$ & $2(4 \%)$ & 34 & & $23(56 \%)$ & $2(4 \%)$ & 25 & \\
\hline Vimentin & & & 43 & 0.021 & & & 41 & 0.031 \\
\hline Negative $(0-10 \%)$ & $33(77 \%)$ & $5(12 \%)$ & 38 & & $23(56 \%)$ & $4(10 \%)$ & 27 & \\
\hline Positive $(>10 \%)$ & $1(2 \%)$ & $4(9 \%)$ & 5 & & $4(10 \%)$ & $10(24 \%)$ & 14 & \\
\hline
\end{tabular}

which is similar to Snail, indicating that Snail and vimentin have a highly positive correlation $(P=0.023$; Table 6$)$.

\section{Discussion}

The malignant potential of gastric cancer has been studied using various EMT regulators such as Sip, Slug, and Snail at the mRNA level [6]. In this study, to examine whether Snail can be a useful marker for the diagnosis of adenocarcinoma, we analyzed the nuclear expression of Snail protein, which is an E-cadherin gene repressor that triggers EMT [1]. As expected, the expression of Snail was significantly higher in gastric adenocarcinomas than in nonneoplastic mucosas or gastric adenomas. Although a few samples of normal gastric tissue and gastritis stained weakly for Snail protein, most benign mucosal samples were negative for Snail. Additionally, in a high percentage of gastric carcinoma biopsies, positive Snail staining was seen in both stromal cells and carcinoma cells. We believe that the stromal cells with positive Snail staining are derived from carcinoma cells in which EMT has occurred although we could not show the direct evidence. In patients with colon cancer, Snail expression in the tumor stroma correlates with worse survival [2]. According to the report by Graham et al. , the cells undergoing EMT in human cancers are usually found on the invasion front of a malignant tumor [5], which is in agreement with our findings from a previous study [8]. However, these techniques were not applicable to the biopsy tissues here, because it was difficult to identify the tumor front.

We observed an inverse relationship between E-cadherin and Snail expression in most of the carcinomas examined, although a small number of neoplastic cells maintained moderate expression in both proteins and did not show evidence of EMT. This result is supported by previous data showing that Snail expression is associated with Ecadherin downregulation in gastric cancer cell lines [9]. During EMT, Snail suppresses the expression of cell adhesion molecules, such as E-cadherin, and increases the expression of mesenchymal cell markers [10]. Vimentin is one of the traditional mesenchymal markers and a major protein constituent of intermediate filaments in normal and neoplastic mesenchymal cells [11]. Our results showed that, 
TABLE 6: Correlation between Snail expression and E-cadherin, vimentin expression in carcinoma biopsy samples.

\begin{tabular}{|c|c|c|c|c|c|c|}
\hline \multirow{2}{*}{ Differentiation } & & \multicolumn{3}{|c|}{ Snail expression in carcinoma } & \multirow{2}{*}{$\begin{array}{c}\text { Total } \\
165\end{array}$} & \multirow[b]{2}{*}{$P$} \\
\hline & & Negative $(<10)$ & Low $(10-20)$ & $\operatorname{High}(>20)$ & & \\
\hline \multirow{6}{*}{ Well } & E-cadherin & & & & 36 & 0.031 \\
\hline & Negative (0-10\%) & 0 & $3(8 \%)$ & $20(56 \%)$ & 23 & \\
\hline & Positive ( $>10 \%)$ & $1(3 \%)$ & $6(16 \%)$ & $6(17 \%)$ & 13 & \\
\hline & Vimentin & & & & 36 & 0.025 \\
\hline & Negative (0-10\%) & $1(3 \%)$ & $4(11 \%)$ & $3(8 \%)$ & 8 & \\
\hline & Positive ( $>10 \%)$ & 0 & $5(14 \%)$ & $23(64 \%)$ & 28 & \\
\hline \multirow{6}{*}{ Moderately } & E-cadherin & & & & 48 & 0.039 \\
\hline & Negative $(0-10 \%)$ & 0 & $5(10 \%)$ & $30(63 \%)$ & 35 & \\
\hline & Positive $(>10 \%)$ & 0 & $8(17 \%)$ & $5(10 \%)$ & 13 & \\
\hline & Vimentin & & & & 48 & 0.032 \\
\hline & Negative $(0-10 \%)$ & 0 & $6(12 \%)$ & $3(6 \%)$ & 9 & \\
\hline & Positive $(>10 \%)$ & 0 & $7(15 \%)$ & $32(67 \%)$ & 39 & \\
\hline \multirow{6}{*}{ Poorly } & E-cadherin & & & & 51 & 0.019 \\
\hline & Negative $(0-10 \%)$ & 0 & $4(8 \%)$ & $41(80 \%)$ & 45 & \\
\hline & Positive (>10\%) & 0 & $3(6 \%)$ & $3(6 \%)$ & 6 & \\
\hline & Vimentin & & & & 51 & 0.023 \\
\hline & Negative $(0-10 \%)$ & 0 & $2(4 \%)$ & $4(8 \%)$ & 6 & \\
\hline & Positive $(>10 \%)$ & 0 & $5(10 \%)$ & $40(78 \%)$ & 45 & \\
\hline \multirow{6}{*}{ Signet-ring cell } & E-cadherin & & & & 30 & 0.042 \\
\hline & Negative $(0-10 \%)$ & $3(10 \%)$ & $5(17 \%)$ & $10(33 \%)$ & 18 & \\
\hline & Positive (>10\%) & $6(20 \%)$ & $5(17 \%)$ & $1(3 \%)$ & 12 & \\
\hline & Vimentin & & & & 30 & 0.044 \\
\hline & Negative (0-10\%) & $7(23 \%)$ & $6(20 \%)$ & 0 & 13 & \\
\hline & Positive $(>10 \%)$ & $2(7 \%)$ & $4(14 \%)$ & $11(36 \%)$ & 17 & \\
\hline
\end{tabular}

among all the samples examined, only adenocarcinomas stained strongly for both Snail and vimentin. These findings are consistent with a report that vimentin is correlated with poor prognosis in cancer patients [12]. However, in our study, Snail staining was observed more specifically than vimentin staining in adenocarcinomas, suggesting that Snail expression is more specific for adenocarcinomas than vimentin.

Gastric cancer remains a national health issue in Japan [13]. Although periodic endoscopic screening can detect early cancer in many cases, improving the prognosis, close cooperation between pathologists and gastroenterologists is still needed for accurate diagnosis. TMN stage is the strongest prognostic factor for survival in gastric cancer [14], but the TMN stage cannot be definitively determined preoperatively. Tumor size, appearance, and depth of penetration in the wall are predictive for lymph node metastasis. These characteristics are estimated based on endoscopic or endosonographic features. According to the treatment guidelines for gastric cancer in Japan, endoscopic treatments (such as endoscopic mucosal resection and endoscopic submucosal dissection) may be offered to patients with well differentiated cancers that are localized in the mucosal layer, less than $2 \mathrm{~cm}$ in diameter, and unaccompanied by ulceration (Japanese Gastric Cancer Association. 2004) [15]. However, due to high rates of local recurrence, more than half of the patients with early gastric cancers remain uncured [16]. In these cases, preoperative evaluation of prognostic factors is important. Some papers have already proposed a new EMT phenotype classification based on the numbers of EMT-related protein changes [17].
We suggest that Snail could be a marker for distinguishing gastric carcinomas from benign tissues. Especially when we encounter a borderline lesion, it would be greatly helpful to decide whether it is benign or malignant. Furthermore, analyzing Snail expression can aid in surgical decision-making because Snail expression promotes tumor invasiveness, migration, and proliferation activity [18], and because most of the carcinomas stained positively for Snail. Therefore, we suggest that Snail expression could be regarded as a novel marker for the diagnosis of gastric cancer and might be used to aid in surgical decision-making in patients with gastric cancer.

\section{Acknowledgments}

The authors would like to thank Mayumi Akira for technical assistance. This work was supported in part by the Ministry of Education, Science, Sports, and Culture of Japan and a Research Grant for Priority Areas from Wakayama Medical University (to Y. Muragaki).

\section{References}

[1] A. Cano, M. A. Pérez-Moreno, I. Rodrigo et al., "The transcription factor Snail controls epithelial-mesenchymal transitions by repressing E-cadherin expression," Nature Cell Biology, vol. 2, no. 2, pp. 76-83, 2000.

[2] C. Franci, M. Gallen, F. Alameda et al., "Snaill protein in the stroma as a new putative prognosis marker for colon tumours," PLoS ONE, vol. 4, no. 5, Article ID e5595, 2009. 
[3] K. F. Becker, E. Rosivatz, K. Blechschmidt, E. Kremmer, M. Sarbia, and H. Höfler, "Analysis of the E-cadherin repressor snail in primary human cancers," Cells Tissues Organs, vol. 185, no. 1-3, pp. 204-212, 2007.

[4] M. Fujii, M. Kochi, and T. Takayama, "Recent advances in chemotherapy for advanced gastric cancer in Japan," Surgery Today, vol. 40, no. 4, pp. 295-300, 2010.

[5] D. Y. Graham, M. Kato, and M. Asaka, "Gastric endoscopy in the 21st century: appropriate use of an invasive procedure in the era of non-invasive testing," Digestive and Liver Disease, vol. 40, no. 7, pp. 497-503, 2008.

[6] C. Castro Alves, E. Rosivatz, C. Schott et al., "Slug is overexpressed in gastric carcinomas and may act synergistically with SIPI and Snail in the down-regulation of E-cadherin," Journal of Pathology, vol. 211, no. 5, pp. 507-515, 2007.

[7] T. Sano and Y. Kodera, "Japanese gastric cancer treatment guidelines 2010 (ver. 3)," Gastric Cancer, vol. 14, no. 2, pp. 113-123, 2011.

[8] R. Nishioka, S. Itoh, T. Gui et al., "SNAIL induces epithelial-tomesenchymal transition in a human pancreatic cancer cell line (BxPC3) and promotes distant metastasis and invasiveness in vivo," Experimental and Molecular Pathology, vol. 89, no. 2, pp. 149-157, 2010.

[9] E. Rosivatz, I. Becker, K. Specht et al., "Differential expression of the epithelial-mesenchymal transition regulators Snail, SIP1, and twist in gastric cancer," American Journal of Pathology, vol. 161, no. 5, pp. 1881-1891, 2002.

[10] C. Pena, J. M. Garcia, M. J. Larriba et al., "SNAI1 expression in colon cancer related with $\mathrm{CDH} 1$ and VDR downregulation in normal adjacent tissue," Oncogene, vol. 28, no. 49, pp. 43754385, 2009.

[11] K. Vuoriluoto, H. Haugen, S. Kiviluoto et al., "Vimentin regulates EMT induction by Slug and oncogenic H-Ras and migration by governing Axl expression in breast cancer," Oncogene, vol. 30, no. 12, pp. 1436-1448, 2011.

[12] K. Takemura, R. Hirayama, K. Hirokawa et al., "Expression of vimentin in gastric cancer: a possible indicator for prognosis," Pathobiology, vol. 62, no. 3, pp. 149-154, 1994.

[13] M. Asaka, M. Kato, and D. Y. Graham, "Strategy for eliminating gastric cancer in Japan," Helicobacter, vol. 15, no. 6, pp. 486-490, 2010.

[14] T. Aoyama, T. Yoshikawa, T. Watanabe et al., "Macroscopic tumor size as an independent prognostic factor for stage II/III gastric cancer patients who underwent D2 gastrectomy followed by adjuvant chemotherapy with S-1," Gastric Cancer, vol. 14, no. 3, pp. 274-278, 2011.

[15] N. Kaibara, Y. Otani, H. Inoue et al., "Meeting report of the 76th Congress of the Japanese Gastric Cancer Association," Gastric Cancer, vol. 7, no. 4, pp. 185-195, 2004.

[16] T. Foukakis, L. Lundell, M. Gubanski, and P. A. Lind, "Advances in the treatment of patients with gastric adenocarcinoma," Acta Oncologica, vol. 46, no. 3, pp. 277-285, 2007.

[17] M. A. Kim, H. S. Lee, H. E. Lee, J. H. Kim, H. K. Yang, and W. H. Kim, "Prognostic importance of epithelial-mesenchymal transition-related protein expression in gastric carcinoma," Histopathology, vol. 54, no. 4, pp. 442-451, 2009.

[18] Y. Usami, S. Satake, F. Nakayama et al., "Snail-associated epithelial-mesenchymal transition promotes oesophageal squamous cell carcinoma motility and progression," Journal of Pathology, vol. 215, no. 3, pp. 330-339, 2008. 


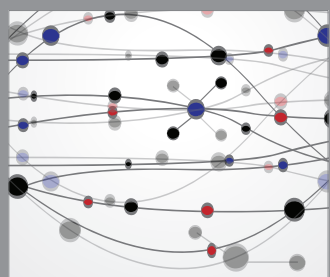

The Scientific World Journal
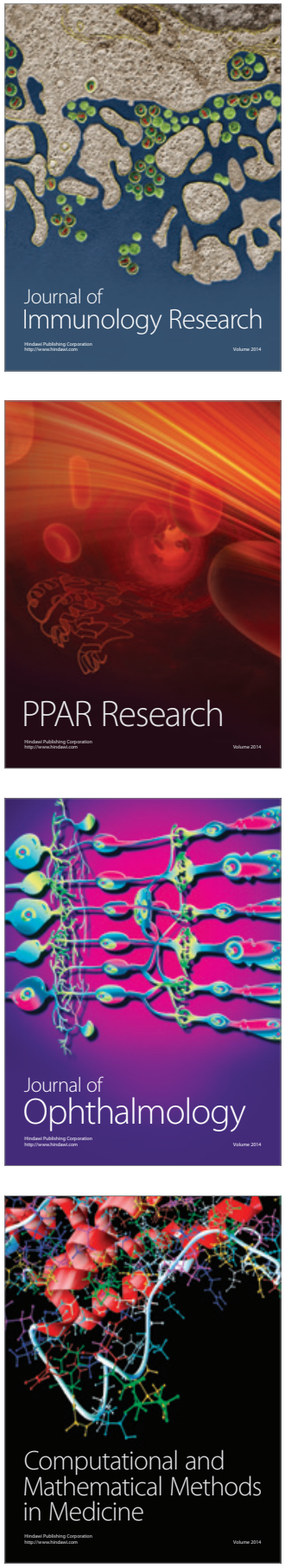

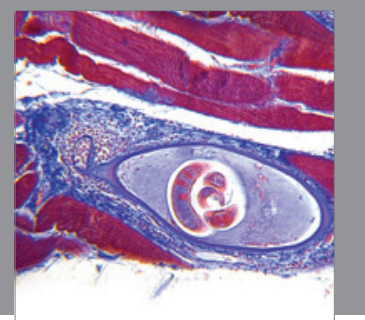

Gastroenterology

Research and Practice
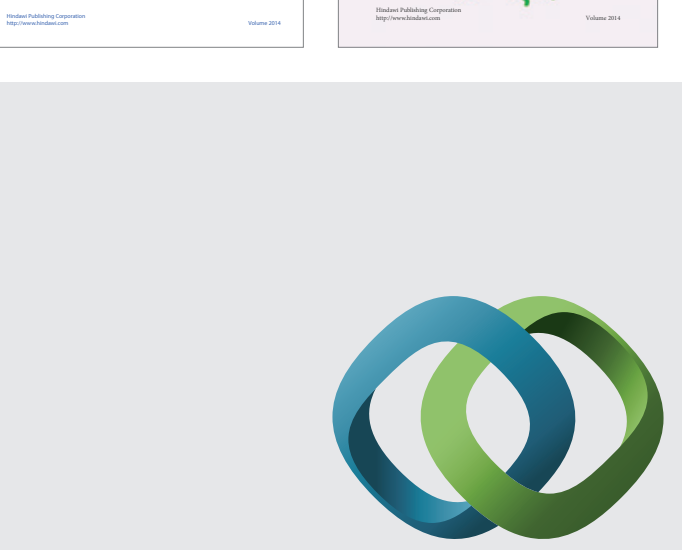

\section{Hindawi}

Submit your manuscripts at

http://www.hindawi.com
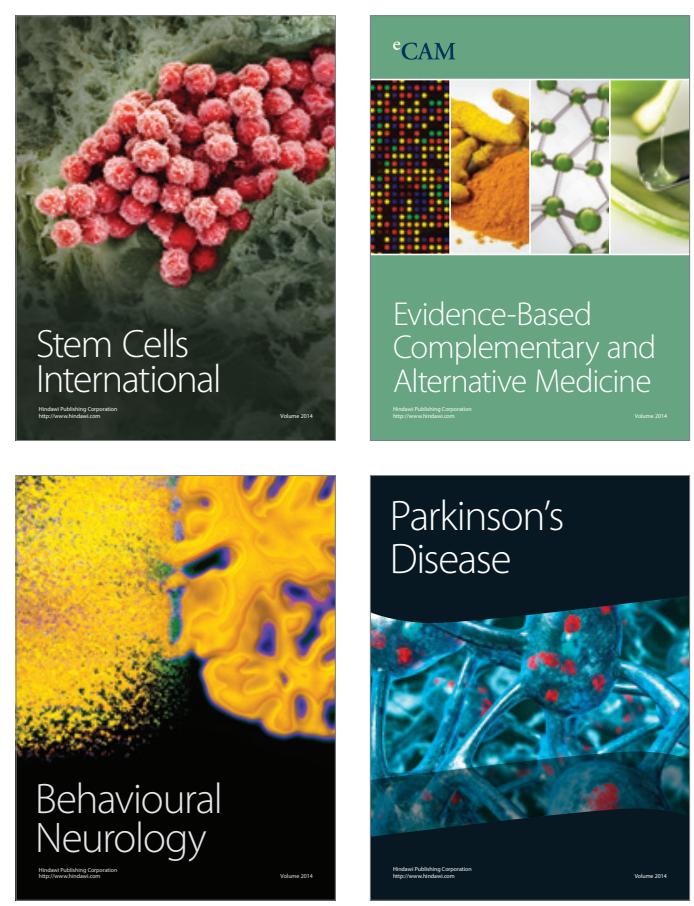

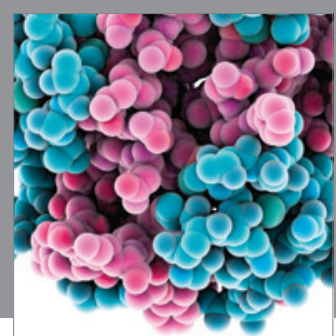

Journal of
Diabetes Research

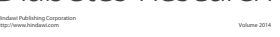

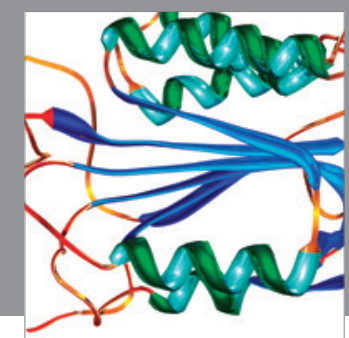

Disease Markers
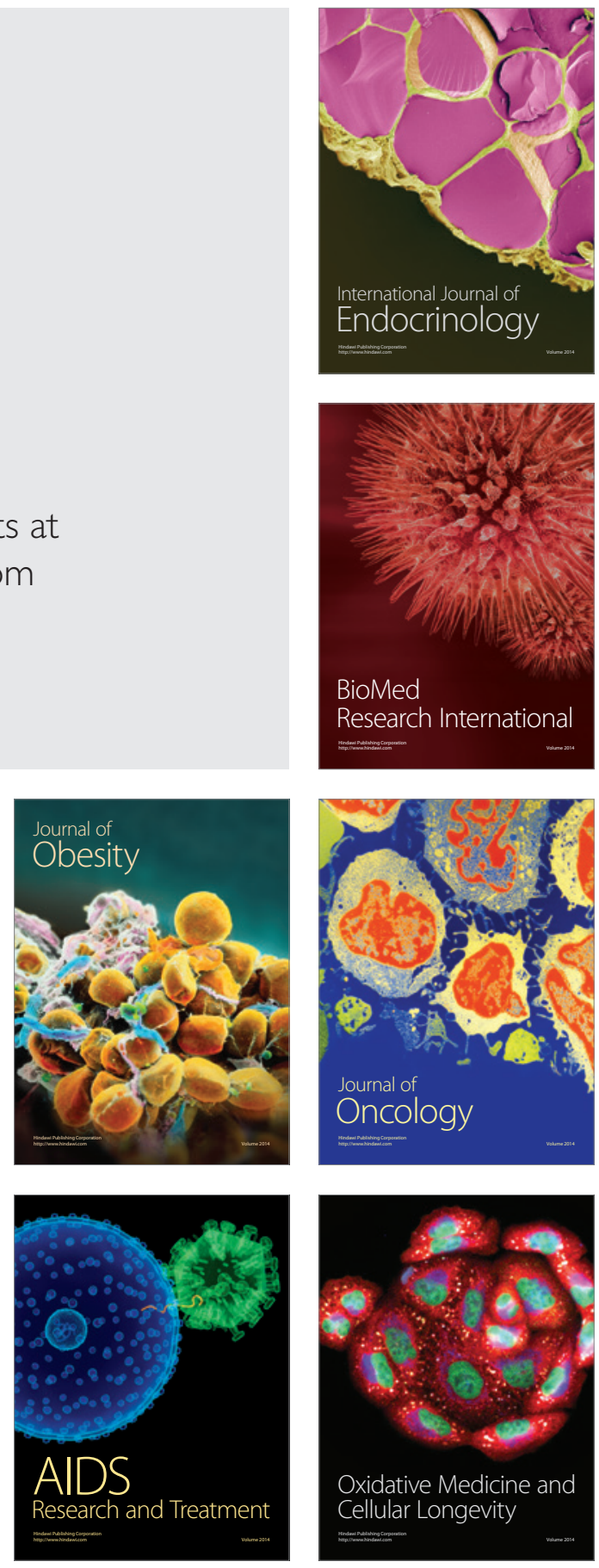